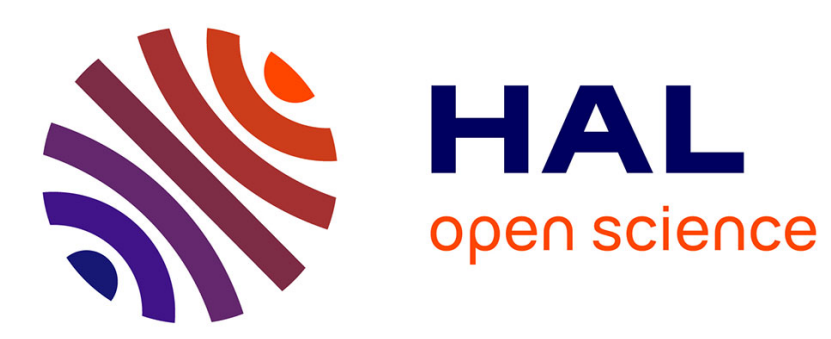

\title{
A Fuzzy Spatio-Temporal-based Approach for Activity Recognition
}

\author{
Jean-Marie Le Yaouanc, Jean-Philippe Poli
}

\section{To cite this version:}

Jean-Marie Le Yaouanc, Jean-Philippe Poli. A Fuzzy Spatio-Temporal-based Approach for Activity Recognition. International Workshop on Semantic and Conceptual Issues in GIS (SeCoGIS 2012), Oct 2012, Florence, Italy. pp.314-324. hal-00739277

\section{HAL Id: hal-00739277 \\ https://hal.science/hal-00739277}

Submitted on 7 Oct 2012

HAL is a multi-disciplinary open access archive for the deposit and dissemination of scientific research documents, whether they are published or not. The documents may come from teaching and research institutions in France or abroad, or from public or private research centers.
L'archive ouverte pluridisciplinaire HAL, est destinée au dépôt et à la diffusion de documents scientifiques de niveau recherche, publiés ou non, émanant des établissements d'enseignement et de recherche français ou étrangers, des laboratoires publics ou privés. 


\title{
A Fuzzy Spatio-Temporal-based Approach for Activity Recognition
}

\author{
Jean-Marie Le Yaouanc and Jean-Philippe Poli \\ CEA, LIST, 91191 Gif-sur-Yvette CEDEX, FRANCE \\ \{firstname.lastname\}@cea.fr
}

\begin{abstract}
Over the last decade, there has been a significant deployment of systems dedicated to surveillance. These systems make use of real-time sensors that generate continuous streams of data. Despite their success in many cases, the increased number of sensors leads to a cognitive overload for the operator in charge of their analysis. However, the context and the application requires an ability to react in real-time. The research presented in this paper introduces a spatio-temporal-based approach the objective of which is to provide a qualitative interpretation of the behavior of an entity (e.g., a human or vehicle). The process is formally supported by a fuzzy logic-based approach, and designed in order to be as generic as possible.
\end{abstract}

Keywords: Spatio-temporal data modeling, Automatic activity recognition, Semantic trajectories, Fuzzy logic.

\section{Introduction}

Surveillance is of growing interest because of the importance of safety and security issues. When integrated with Geographical Information Systems (GIS), systems dedicated to surveillance combine spatial features with the information provided by real-time sensors to provide a support for the management of entities (e.g., humans or vehicles). Supervision of mobile entities has a wide range of potential applications, such as the security and safety of critical buildings (e.g. stadiums, airports), or the traffic surveillance in cities. However, the increase use of sensors leads to a saturation for the human operator in charge of the data analysis. Consequently, it would be desirable to develop systems that assist humans in supervising spatial scenes, i.e. systems that automatically analyze data streams, detect suspicious events, and advise an operator to check a particular screen.

Automatic activity recognition is a process the objective of which is to interpret the behavior of entities in order to generate a description of the detected events or to raise an alarm. The capture of information associated to these entities is operated by sensors such as video cameras that collect images of a specific environment, or geo-positioning systems that record geographical positions. Using time intervals and logical formalisms, previous approaches have obtained 
successful results in detecting high level activities 1]. Formal rules have been defined for detecting activities involving interactions amongst people or recognize unusual behaviors of individuals $[2,3$. More recently, an expert system was used to combine facts detected by a low-level framework, and inference rules previously defined by an expert 4]. Petri nets have also been considered as a well-adapted representation and recognition support [5]. F. Bremond rather suggests the use of finite state machines, where states represent sub-activities, and transitions, the events. An activity is recognized if its final state is reached 6. Hereafter, we focus on the automatic recognition of activities from the interpretation of trajectories. Spatio-temporal configurations between two mobile entities can be detected by analyzing their relative distances and speeds 7,8 . B. Gottfried defines a spatio-temporal model based on the analysis of the evolution of relative directions between two mobile entities 9 . Other models are specifically designed for spatial databases, and particular operators are defined that optimize the implementation of complex spatio-temporal queries 10,11 . However, these approaches have limitations in handling uncertainties and variations since they identify activities only when their spatial and temporal relationships are strictly satisfied, ignoring the variations. As a matter of fact, the execution of an event is usually dependent of the context and the intrinsic characteristics of the entity.

The research presented in this paper concerns the real-time semantic interpretation of the behavior of a mobile entity observed by sensors. Real-time sensors generate a huge amount of quantitative data. However, these data do not completely reflect the way a human perceives and describes an environment since he preferably stores and processes qualitative information. As a consequence, we provide a semantic model suitable with cognition, but also appropriated for the processing of spatio-temporal data. The model analyses the quantitative data recorded by sensors and evaluates behaviors involving entities (i.e. humans or vehicles). Since it is designed to consider uncertainties of the activities' structures, the qualitative interpretation is supported by a fuzzy-based approach that provides a fuzzy interpretation of the spatial and temporal dimensions.

The reminder of the paper is organized as follows. Section 2 briefly introduces basic principles on fuzzy logic. Section 3 provides a conceptual representation of an activity and models fuzzy spatio-temporal relations. Finally, Section 4 draws the conclusions and outlines further work.

\section{Basic principles on fuzzy logic}

Fuzzy logic was designed to allow systems to mimic the way humans think. Fuzzy logic is based upon the fuzzy set theory that is a formal mathematical theory dedicated to the representation of uncertainty [12. The approach is particularly relevant when dealing with real world systems that interact with humans, since humans mainly manipulate qualitative information. Hereafter, we briefly outline how fuzzy logic extends classical logic. Let us denote $X$ a universe of discourse, 
a fuzzy subset $A \subset X$ is characterized by its membership function $\mu_{A}$,

$$
\mu_{A}: X \rightarrow[0,1]
$$

For each $x \in X$, the value $\mu_{A}(x)$ is interpreted as the degree of membership of $x$ in the fuzzy set $A$, or, equivalently, as the truth value of the proposition "x is an element of A". In order to generalize the set theoretical operations intersections and unions, triangular norms (t-norms) and conorms (t-conorms) were defined. Although there are many ways to define t-norms and t-conorms, only few are used in applications. One of the most used t-norm, together with its dual t-conorm is the one defined by L. Zadeh: $x \wedge y=\min (x, y), x \vee y=\max (x, y)$.

\section{$3 \quad$ Modeling approach}

\subsection{Conceptual modeling of an activity}

We model an activity by a Situation Graph Tree (SGT) [13], the objective of which is to facilitate the understanding of the structures that emerge from the description of an activity. SGT are hierarchical trees that characterize the behavior of entities in terms of situations they can be in. Such a graph illustrates the combination of elementary units that model a particular situation with hierarchical (e.g., a situation composed of several sub-situations), temporal (e.g., a situation that occurs before or while another one) and semantic relations. As a matter of fact, the semantics related to an entity at a given time is contained in an elementary unit, which constitutes the basic component of a SGT. Elementary units that represent different temporal episodes of the same situation are enclosed by the situation graph. We characterize an elementary unit as a semantic function that qualitatively evaluates a situation or an action. An elementary unit relates an entity (that may be dynamic or static) with a spatial object, i.e. a landmark or a form of the environment. Landmarks are salient objects that structure a cognitive representation of an environment 14 . They constitute keyreferences for the conceptualization and the description of an environment, and consequently play a prominent role for describing a spatial situation or characterizing the movement of an entity in an environment. The principles of the modeling approach being introduced, we hereafter present the formal representation of an elementary unit. Let $\mathbb{G}$ be the set of SGT, $\mathbb{U}$ the set of elementary units composing a SGT, $\mathbb{E}$ the set of mobile entities, $\mathbb{R}$ the set of spatio-temporal relations and $\mathbb{O}$ the set of simple spatial objects, i.e., landmarks or forms that structure an environment. A situational graph tree $\mathcal{G} \in \mathbb{G}$ is an ordered set of elementary units $u_{i} \in \mathbb{U}$, i.e., $\mathcal{G}=\left[u_{1}, \ldots, u_{n}\right]$ where $n \geq 1$. An elementary unit $u_{i}$ is a triplet such as $u_{i}=\left[e_{j}, r_{k}, o_{l}\right]$ with $e_{j} \in \mathbb{E}, r_{k} \in \mathbb{R}$ and $o_{l} \in \mathbb{O}$.

\subsection{Modeling of spatio-temporal relations}

In the following subsections, the modeling of spatio-temporal relations that characterize the activity of a mobile entity in an environment is developed. In order 
to consider the uncertainties of the activities' structures, the semantic interpretation of the entity's trajectory is supported by a fuzzy-based approach. It is designed to be as generic as possible, and considers objects with rather bona fide or fiat boundaries. The former are objects with physical discontinuities (e.g. a mountain or a valley), the latter gets boundaries induced through human demarcation (e.g. a building or an administrative region) [15]. The development of formal models of topological relations has received much attention in the literature of GIS, computer vision and image understanding [16]. In recent years, significant achievement have been made on the development of formal models of topological relations between spatial objects with indeterminate boundaries. C. Hudelot and I. Bloch defined spatial relations such as the adjacency and inclusion, but also directional relations between fuzzy image regions [17]. Among the GIS community, E. Clementini and P. Di Felice [18], and A. Cohn and N. Gotts 19] developed models of topological relations between fuzzy regions, e.g. Disjoint, Meet or Inside.

Temporal representation and reasoning is also an important facet in the design of a fuzzy spatio-temporal approach. As a matter of fact, when the time span of an activity is imprecise, it can be represented by a fuzzy time interval. J. F. Allen defined a set of 13 qualitative relations, e.g. Before and After, that may hold between two intervals [20], and his work was recently extended to a more general formalism that can handle precise as well as imprecise relationships between crisp and fuzzy intervals 21]. P. Cariñena provides a complementary approach and models the temporal relations Occurrence and Persistence between an event and a fuzzy temporal interval 22 .

Let $\mathbb{I}$ be the set of temporal intervals, $\mathbb{T}$ the set of instants, $\mathbb{O}$ the set of simple spatial objects, $\mathbb{O}_{1} \subset \mathbb{O}$ the set of simple closed regions, $\mathbb{O}_{2} \subset \mathbb{O}$ the set of simple opened regions, $\mathbb{P}$ the set of fuzzy propositions and $\mathbb{F}$ the set of fuzzy membership functions. Let $p \in \mathbb{P}$ be a fuzzy proposition, and $\mu(p, t)$ the value of $p$ at a given moment $t$. Let $I \in \mathbb{I}$ be a temporal interval and $t$ a given moment. We denote $I^{*}=I \backslash\{t\}$. Let Mean be the function that computes the mean of a set of fuzzy values along a given interval $I$ :

$$
\text { Mean } \begin{aligned}
\mathcal{F} \times \mathbb{I} & \rightarrow \mathcal{F} \text {, with } \mathcal{F} \text { the set of fuzzy values } \\
(\mu(p, t), I) & \mapsto \frac{\sum_{t \in I} \mu\left(p, t_{i}\right)}{\operatorname{Card}(t)}, \text { where } \operatorname{Card}() \text { is the cardinality operator. }
\end{aligned}
$$

Relation IsMoving The spatio-temporal relation IsMoving characterizes the moving of a mobile entity in a non-constraint space 11 Its evaluation takes into account the positions of the considered entity during a past time interval. It is based on the assumption that the value at time $t_{i}$ may not only be based on the last moving between $t_{i-1}$ and $t_{i}$, but on their recording in the past. Consequently, if the entity $e$ is not moving between times $t_{i-1}$ and $t_{i}$, the value of the relation IsMoving is pondered by its previous moving during a given past

\footnotetext{
${ }^{1}$ The relation IsMoving relates an entity to the studied environment. To facilitate the reading, this object is not clearly mentioned.
} 
time interval. In other words, if a pedestrian stops walking at time $t$ because he is looking for his keys, the value of the relation IsMoving will decrease in time if he stops during a significant time. More formally, let $I_{1} \in \mathbb{I}, p_{1} \in \mathbb{P}$ be the fuzzy proposition "the distance travelled by $e$ is not zero". The fuzzy proposition $p_{1}$ is correlated to the moving distance of $e$ between instants $t_{i-1}$ and $t_{i}$. Figure 1 illustrates a possible representation of $f_{1}$, the fuzzy membership of $p_{1}$. Let IsMoving() be the function that models the moving of an entity,

$$
\begin{aligned}
\text { IsMoving : } \mathbb{E} \times \mathbb{F} \times \mathbb{I} \times \mathbb{T} & \rightarrow \mathcal{F}, \text { with } \mathcal{F} \text { the set of fuzzy values } \\
\left(e, f_{1}, I_{1}, t\right) & \mapsto \mu\left(p_{1}, t\right) \vee \operatorname{Mean}\left(\mu\left(p_{1}, t\right), I_{1}^{*}\right) .
\end{aligned}
$$

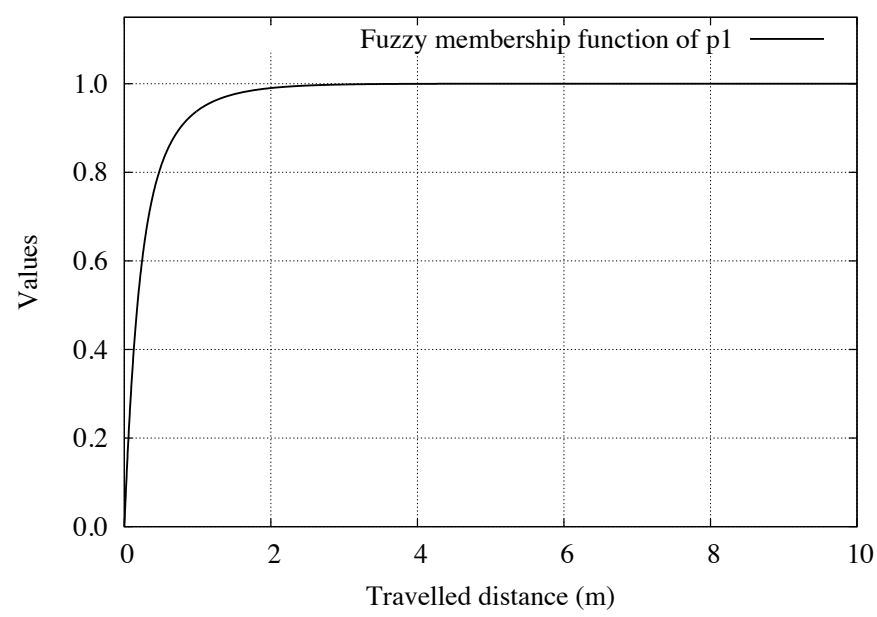

Fig. 1. Fuzzy membership function $f_{1}$

Relation IsComingClose To The spatio-temporal relation IsComingCloseTo characterizes the approach of a spatial object $o \in \mathbb{O}$ by a mobile entity $e \in \mathbb{E}$ in a non-constraint space. For instance, this relation may be useful for characterizing a boat that is coming close to a navigational buoy. Intuitively, the closer the entity to $o$ in a way that minimizes the distance to reach the object, the higher the fuzzy value of IsComingClose To is. Two spatial configurations are defined, with respect to the geometry of $o$.

We study the approach of an object modelled as a point or an open polyline. The geometry of the given object is provided by a vector geo-database that covers the studied environment. Let $P\left(t_{i}\right)$ be the position of $e$ at time $t_{i}, N\left(t_{i}\right)$ the point that minimizes the distance between $e$ and $o$ at time $t_{i}$, and $\overline{P\left(t_{i}\right) Q\left(t_{i}\right)}$ the vector that identifies the direction of $e$ at time $t_{i}$ (Fig. 2). The evaluation of the relation takes into account:

- the evolution of the location of $e$ between times $t_{i}$ and $t_{i-1}$.

- the orientation $\alpha\left(t_{i}\right)=\left(\overrightarrow{P\left(t_{i}\right) N\left(t_{i}\right)}, \overrightarrow{P\left(t_{i}\right) Q\left(t_{i}\right)}\right)$ of $e$ at time $t_{i}$. Thus, the more $\cos \left(\alpha\left(t_{i}\right)\right)$ tends to 1 , the higher the value of IsComingCloseTo. 
- the recording of orientations $\alpha\left(t_{i}\right)$ during a temporal interval $I^{*} \in \mathbb{I}$. Thus, the more Mean $\left(\max \left(\cos \left(\alpha\left(t_{i}\right)\right), 0\right), I^{*}\right)$ tends to 1 , the higher the value of IsComingCloseTo.

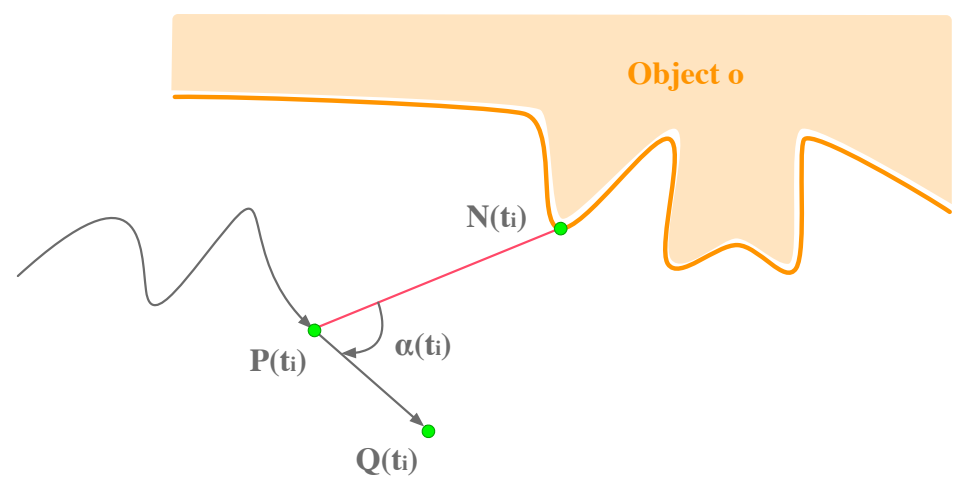

Fig. 2. Approach of an object modelled as a point or an open polyline

Let $I_{1} \in \mathbb{I}, p_{2} \in \mathbb{P}$ be the fuzzy proposition "the value of $\max \left(\cos \left(\max \left(\alpha\left(t_{i}\right), 0\right)\right), 0\right)$ tends to $1 "$ and $f_{2} \in \mathbb{F}$ its fuzzy membership. Figure 3 illustrates a possible representation of $f_{2}$, the fuzzy membership of $p_{2}$.

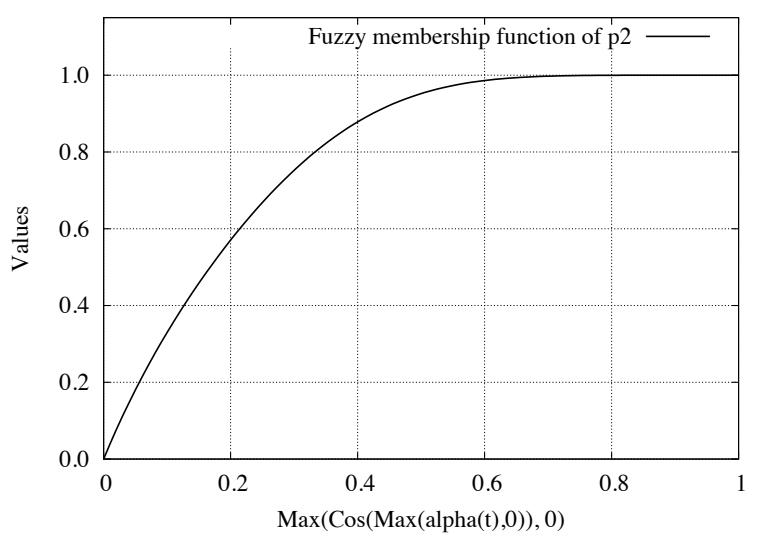

Fig. 3. Fuzzy membership function $f_{2}$

Let us denote IsComingCloseTo() the function that models the approach of an entity,

IsComingCloseTo: $\mathbb{E} \times \mathbb{O}_{2} \times \mathbb{F}^{2} \times \mathbb{I} \times \mathbb{T} \rightarrow \mathcal{F}$, with $\mathcal{F}$ the set of fuzzy values $\left(e, o, f_{1}, f_{2}, I_{1}, t\right) \mapsto \operatorname{IsMoving}\left(e, f_{1}, I_{1}, t\right)$ $\wedge\left(\mu\left(p_{2}, t\right) \vee \operatorname{Mean}\left(\mu\left(p_{2}, t\right), I_{1}^{*}\right)\right)$ 
We consider now the approach of an object modelled as a closed region. Let $P\left(t_{i}\right)$ be the position of $e$ at time $t_{i}, \Delta_{1}$ and $\Delta_{2}$ the exterior tangents of object $o \in \mathbb{O}_{1}$ that pass through the point $P\left(t_{i}\right), P_{\Delta_{1}}\left(t_{i}\right)$ and $P_{\Delta_{2}}\left(t_{i}\right)$ the tangent points to $o$ and the tangents, $\left(P\left(t_{i}\right) M\left(t_{i}\right)\right)$ the median line that bisects the angle $\left(\overrightarrow{P\left(t_{i}\right) P_{\Delta_{1}}\left(t_{i}\right)}, \overrightarrow{P\left(t_{i}\right) P_{\Delta_{2}}\left(t_{i}\right)}\right)$, and $\overrightarrow{P\left(t_{i}\right) Q\left(t_{i}\right)}$ the vector that identifies the direction of $e$ at time $t_{i}$. Let us denote $\beta\left(t_{i}\right)=\left(\overrightarrow{P(t) M\left(t_{i}\right)}, \overrightarrow{P\left(t_{i}\right) P_{\Delta_{1}}\left(t_{i}\right)}\right)$ and $\alpha\left(t_{i}\right)=\left(\overrightarrow{P\left(t_{i}\right) M\left(t_{i}\right)}, \overrightarrow{P\left(t_{i}\right) Q\left(t_{i}\right)}\right)$ (Fig. 4). Intuitively, the lesser the value of angle $\alpha\left(t_{i}\right)$, the higher the value of the relation. However, as soon as the direction of $e$ is inside in the directional cone $\left(\overrightarrow{P\left(t_{i}\right) P_{\Delta_{2}}\left(t_{i}\right)}, \overrightarrow{P\left(t_{i}\right) P_{\Delta_{1}}\left(t_{i}\right)}\right)$ (represented in grey in Figure 4), the relation gets the maximal fuzzy value, i.e., 1.

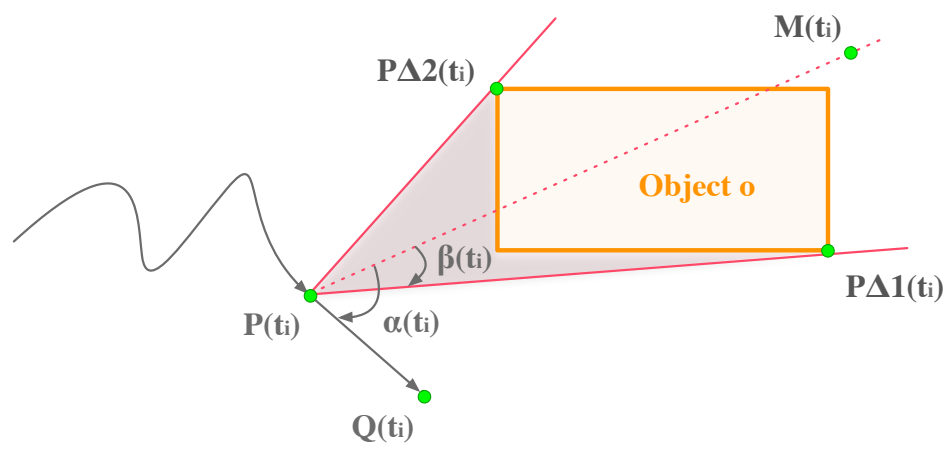

Fig. 4. Approach of an object modelled as a closed region

The evaluation of the relation takes into account:

- the location of $e$ relatively to $o$

- the evolution of the location of $e$ between times $t_{i}$ and $t_{i-1}$.

- the orientation $\alpha\left(t_{i}\right)$ of entity $e$ at time $t_{i}$. Thus, the more $\cos \left(\max \left(\alpha\left(t_{i}\right)-\right.\right.$ $\left.\left.\beta\left(t_{i}\right), 0\right)\right)$ tends to 1 , the higher the value of IsComingClose To is.

- the recording of orientations $\alpha\left(t_{i}\right)$ during a temporal interval $I^{*} \in \mathbb{I}$. Thus, the more $\operatorname{Mean}\left(\max \left(\cos \left(\max \left(\alpha\left(t_{i}\right)-\beta\left(t_{i}\right), 0\right)\right), 0\right), I^{*}\right)$ tends to 1 , the higher the value of IsComingCloseTo.

Let $I_{1} \in \mathbb{I}, p_{3} \in \mathbb{P}$ the fuzzy proposition "the value of $\max \left(\cos \left(\max \left(\alpha\left(t_{i}\right)-\right.\right.\right.$ $\left.\left.\left.\beta\left(t_{i}\right), 0\right)\right), 0\right)$ tends to $1 "$ and $f_{3} \in \mathbb{F}$ its fuzzy membership. Let us denote IsComingCloseTo() the function that models the approach of an object,

$$
\begin{aligned}
\text { IsComingCloseTo: }: \mathbb{E} \times \mathbb{O}_{1} \times \mathbb{F}^{2} \times \mathbb{I} \times \mathbb{T} \rightarrow & \mathcal{F}, \text { with } \mathcal{F} \text { the set of fuzzy values } \\
\left(e, o, f_{1}, f_{3}, I_{1}, t\right) \mapsto & \mu\left(\text { Persistence }\left(\operatorname{Disjoint}(e, o), I_{1}\right)\right) \\
& \wedge I \operatorname{Is} \operatorname{Moving}\left(e, f_{1}, I_{1}, t\right) \\
& \wedge\left(\mu\left(p_{3}, t\right) \vee \operatorname{Mean}\left(\mu\left(p_{3}, t\right), I_{1}^{*}\right)\right)
\end{aligned}
$$

Relation IsGoingAway The spatio-temporal relation IsGoingAway characterizes the moving away of a mobile entity $e \in \mathbb{E}$ from a spatial object $o \in \mathbb{O}$ in a 
non-constraint space. For instance, this relation may be useful for characterizing a boat that moves away for the coast. Intuitively, the more the entity moves away from $o$ in a way that maximize the distance to reach the object, the higher the fuzzy value of IsGoingAway. Two spatial configurations that are similar to the case illustrated in Section 3.2 are defined, with respect to the geometry of $o$.

We study the approach of an object modelled as a point or an open polyline. The evaluation of the relation takes into account:

- the evolution of the location of $e$ between times $t_{i}$ and $t_{i-1}$.

- the orientation $\alpha\left(t_{i}\right)$ of entity $e$ at time $t_{i}$. Thus, the more $\cos \left(\alpha\left(t_{i}\right)\right)$ tends to -1 , the higher the value of Is GoingAway.

- the recording of orientations $\alpha\left(t_{i}\right)$ during a temporal interval $I^{*} \in \mathbb{I}$. Thus, the more Mean $\left(\min \left(\cos \left(\alpha\left(t_{i}\right)\right), 0\right), I^{*}\right)$ tends to -1 , the higher the value of IsGoingAway.

Let $I_{1} \in \mathbb{I}, p_{4} \in \mathbb{P}$ the fuzzy proposition "the value of $\min \left(\cos \left(\alpha\left(t_{i}\right)\right), 0\right)$ tends to -1 " and $f_{4} \in \mathbb{F}$ its fuzzy membership. Let us denote IsGoingAway() the function that models the moving away from an object,

$$
\begin{aligned}
\text { IsGoing Away : } \mathbb{E} \times \mathbb{O}_{2} \times \mathbb{F}^{2} \times \mathbb{I} \times \mathbb{T} & \rightarrow \mathcal{F} \text {, with } \mathcal{F} \text { the set of fuzzy values } \\
\left(e, o, f_{1}, f_{4}, I_{1}, t\right) \quad \mapsto & \operatorname{IsMoving}\left(e, f_{1}, I_{1}, t\right) \\
& \wedge\left(\mu\left(p_{4}, t\right) \vee \operatorname{Mean}\left(\mu\left(p_{4}, t\right), I_{1}^{*}\right)\right)
\end{aligned}
$$

Hereafter, we study the approach of an object modelled as a closed region. Figure 4 illustrates the considered spatial configuration. The evaluation of the relation takes into account:

- the location of $e$ relatively to $o$

- the evolution of the location of $e$ between times $t_{i}$ and $t_{i-1}$.

- the orientation $\alpha\left(t_{i}\right)$ of entity $e$ at time $t_{i}$. Thus, the more $\cos \left(\max \left(\alpha\left(t_{i}\right)-\right.\right.$ $\left.\beta\left(t_{i}\right), 0\right)$ ) tends to -1 , the higher the value of Is GoingAway.

- the recording of orientations $\alpha\left(t_{i}\right)$ during a temporal interval $I^{*} \in \mathbb{I}$. Thus, the more $\operatorname{Mean}\left(\min \left(\cos \left(\max \left(0, \alpha\left(t_{i}\right)-\beta\left(t_{i}\right)\right)\right), 0\right), I^{*}\right)$ tends to -1 , the higher the value of IsGoingAway.

Let $I_{1} \in \mathbb{I}, p_{5} \in \mathbb{P}$ the fuzzy proposition "the value of $\min \left(\cos \left(\max \left(0, \alpha\left(t_{i}\right)-\right.\right.\right.$ $\left.\left.\left.\beta\left(t_{i}\right)\right)\right), 0\right)$ tends to -1 " and $f_{5} \in \mathbb{F}$ its fuzzy membership. Let us denote IsGoingAway() the function that models the moving away from an object,

$$
\begin{aligned}
\text { IsGoing Away : } \mathbb{E} \times \mathbb{O}_{1} \times \mathbb{F}^{2} \times \mathbb{I} \times \mathbb{T} \rightarrow & \mathcal{F} \text {, with } \mathcal{F} \text { the set of fuzzy values } \\
\left(e, o, f_{1}, f_{4}, I_{1}, t\right) \mapsto & \mu\left(\text { Persistence }\left(\operatorname{Disjoint}(e, o), I_{1}\right)\right) \\
& \wedge I \operatorname{Is} \operatorname{Moving}\left(e, f_{1}, I_{1}, t\right) \\
& \wedge\left(\mu\left(p_{5}, t\right) \vee \operatorname{Mean}\left(\mu\left(p_{5}, t\right), I_{1}^{*}\right)\right)
\end{aligned}
$$

Relation IsGoingAlong The spatio-temporal relation IsGoingAlong characterizes the action of an entity $e \in \mathbb{E}$ that goes along a spatial object $o \in \mathbb{O}$ in a non-constraint space. For instance, this relation may be useful for characterizing a boat that sails along the coast. Intuitively, the more the entity significantly moves close to $o$, the higher the fuzzy value of IsGoingAlong. The evaluation of the relation takes into account: 
- the evolution of the location of $e$ between times $t_{i}$ and $t_{i-1}$.

- the proximity of $e$ to $o$ during a significant time span.

Let $I_{1} \in \mathbb{I}, p_{6} \in \mathbb{P}$ the fuzzy proposition "the entity $e$ is near the object $o$ " and $f_{6} \in \mathbb{F}$ its fuzzy membership. Let us denote IsGoingAlong() the function that models the action of going along an object,

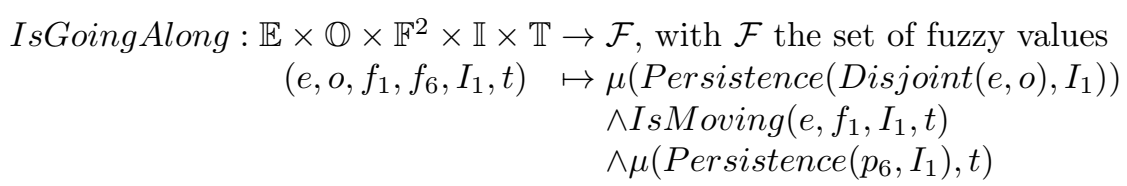

\section{Conclusion}

Current systems dedicated to automatic activity recognition do not consider the spatial and temporal uncertainties, and identify particular activities only when spatial and temporal relationships are strictly satisfied. However, the context and the environment may influence the behavior of a mobile entity. The research presented in this paper introduces an approach for qualifying the activities of a mobile entity in real time. It analyses the trajectory of mobile entities recorded by sensors and qualitatively evaluates their behavior. It is supported by a fuzzy-based approach that both provides a fuzzy interpretation of the spatial and temporal dimensions. We have designed four spatio-temporal relations that relate an entity to an object of the environment that may get bona fide or fiat boundaries. The approach is currently being implemented and evaluated. The spatial extension of our prototype is based upon DotSpatial, i.e. a .Net library that favors the integration of geographic data and spatial analysis.

Although experienced for elementary activities, the semantic approach may be applied to high level activities. Such a work may be assessed with the use of a fuzzy expert system. Further theoretical work concerns an extension of the ontological background of the approach and the development of complementary spatio-temporal relations, e.g., IsGoingThrough, IsEntering, IsGoingOut and IsFollowingARoute.

Acknowledgements. This work was part of a collaboration between the CEA-LIST and EGIDIUM Technologies.

\section{References}

1. Allen, J.F., Ferguson, G.: Actions and events in interval temporal logic. Journal of Logic and Computation 4(5) (2010) 531-579

2. Shet, V., Harwood, D., Davis, L.: VidMAP: Video monitoring of activity with Prolog. In: IEEE International Conference on Advanced Video and Signal based Surveillance, Como, Italy, IEEE Computer Society (2005) 224-229

3. Geerinck, T., Enescu, V., Ravyse, I., Sahli, H.: Rule-based video interpretation framework: Application to automated surveillance. In: Proceedings of the 5th International Conference on Image and Graphics, Washington, DC, USA, IEEE Computer Society (2009) 341-348 
4. Krausz, B., Herpers, R.: Metrosurv: Detecting events in subway stations. Multimedia Tools and Applications 50(1) (2010) 123-147

5. Ghanem, N., Dementhon, D., Doermann, D., Davis, L.: Representation and recognition of events in surveillance video using petri nets. In: Proceedings of the Conference on Computer Vision and Pattern Recognition Workshops, Washington, DC, USA, IEEE Computer Society (2004)

6. Bremond, F., Medioni, G.: Scenario recognition in airborne video imagery. In: Proceedings of the Conference on Computer Vision and Pattern Recognition Workshops, Santa Barbara, CA, USA, IEEE Computer Society (1998)

7. Van de Weghe, N., Cohn, A., Maeyer, P., Witlox, F.: Representing moving objects in computer-based expert systems: the overtake event example. Expert Systems with Applications 29 (2005) 977-983

8. Noyon, V., Claramunt, C., Devogele, T.: A relative representation of trajectories in geographical spaces. GeoInformatica 11(4) (2007) 479-496

9. Gottfried, B.: Interpreting motion events of pairs of moving objects. GeoInformatica 15(2) (2011) 247-271

10. Erwig, M.: Toward spatiotemporal patterns. Spatio-Temporal Databases 1 (2004) $29-54$

11. Hornsby, K.S., King, K.: Modeling motion relations for moving objects on road networks. GeoInformatica 12(4) (2008) 477-495

12. Zadeh, L.A.: Fuzzy sets. Information and Control 8(3) (1965) 338-353

13. Arens, M., Nagel, H.H.: Behavioral knowledge representation for the understanding and creation of video sequences. In Günter, A., Kruse, R., Neumann, B., eds.: Proceedings of the 26th German Conference on Artificial Intelligence. LNCS, Springer Heidelberg (2003) 149-163

14. Lynch, K.: The Image of the City. The MIT Press, Boston (1960)

15. Smith, B., Varzi, A.: Fiat and bona fide boundaries: Towards an ontology of spatially extended objects. In Hirtle, S., Frank, A., eds.: Spatial Information Theory A Theoretical Basis for GIS. Volume 1329 of LNCS., Springer Heidelberg (1997) 103-119

16. Zhan, F.B.: Approximate analysis of binary topological relations between geographic regions with indeterminate boundaries. Soft Computing - A Fusion of Foundations, Methodologies and Applications 2 (1998) 28-34

17. Hudelot, C., Atif, J., Bloch, I.: Fuzzy spatial relation ontology for image interpretation. Fuzzy Sets Systems 159(15) (2008) 1929-1951

18. Clementini, E., Felice, P.D.: Approximate topological relations. International Journal of Approximate Reasoning 16(2) (1997) 173-204

19. Cohn, A., Gotts, N.: The 'egg-yolk' representation of regions with indeterminate boundaries. In Burrough, P., Frank, A.M., eds.: Specialist Meeting on Spatial Objects with Undetermined Boundaries, Taylor \& Francis (1997) 171-187

20. Allen, J.F.: Maintaining knowledge about temporal intervals. Communication of the ACM 26(11) (1983) 832-843

21. Schockaert, S., Cock, M.D., Kerre, E.E.: Fuzzifying Allen's temporal interval relations. IEEE Transactions on Fuzzy Systems 16(2) (2008) 517-533

22. Cariñena, P., Bugarin, A., Mucientes, M., Barro, S.: A language for expressing fuzzy temporal rules. Mathware \& Soft Computing 7 (2000) 213-227 